\title{
Post-mortem arteriography of cerebral arteriovenous malformations
}

\author{
R. A. RODDA AND G. D. CALVERT \\ From the Departments of Pathology, University of Tasmania, Hobart, Australia, and University of Otago, \\ Dunedin, New Zealand
}

Post-mortem arteriography has been used to show interarterial anastomoses in the leptomeninges (Vander Eecken and Adams, 1953) and pathological cerebral arterial patterns in infarcts (Hutchinson and Yates, 1957; Stein, McCormick, Rodriguez, and Taveras, 1962; Rodda and Dugdale, 1963), in hypertension (Russell, 1963), and in cerebral haemorrhage (Johnson and Yates, 1956; Cole and Yates, 1967), but cerebral arteriovenous anastomoses have been seldom demonstrated (Kaplan, Aronson, and Browder, 1961).

The pattern of the arteriovenous anastomosis may comprise a complex mass of anomalous vessels of almost any calibre, a relatively simple connection between the arterial and venous sides of the cerebral circulation, or a tiny clump of dilated thin-walled channels. The anastomosis may be easily recognized in living arteriograms or at operation when the vessels involved are engorged, but at necropsy when the vessels are collapsed or perhaps disrupted by massive haemorrhage the lesions may be less obvious.

In this report two cases of cerebral arteriovenous malformation are presented in which the vascular pattern of the anastomoses was demonstrated by post-mortem arteriography using a warm-setting gelatin-based radiopaque medium injected into the carotico-vertebral arterial system at simulated blood pressure with a multiple nozzle air piston syringe. The details of the technique have been described previously (Rodda and Dugdale, 1963). The Schlesinger-type injection medium normally remains confined to the arterial side of the circulation and fills arterioles of 40 to $50 \mu$ in diameter, although vessels as small as 10 to $20 \mu$ in diameter may sometimes contain medium. In the two cases described below, the injection medium passed through the arteriovenous anastomoses and was present in massive quantities in the intracranial venous sinuses.

CASE 1

I.O., a 32-year-old woman, was admitted to the Dunedin
Hospital (13291) when four months pregnant with a subarachnoid haemorrhage confirmed by lumbar puncture. A slight weakness of the left face was noted. A hysterotomy and tubal ligation were done. During the next 12 years she had five or six grand mal epileptic attacks which were without any localizing features. She also complained frequently of headaches sometimes on the right and sometimes on the left side.

At the age of $\mathbf{4 4}$ she was admitted semi-comatose to the Oamaru Hospital with a diagnosis of subarachnoid haemorrhage. She had some weakness of the left side of her face and increased tone in the left arm and the left leg. The lumbar cerebrospinal fluid was heavily bloodstained. Dr. A. C. Begg reported that commono carotid arteriography showed a large, superficial arteriovenous malformation in the lower right parietal region fed by a considerably enlarged middle cerebral artery and drained by a series of very large superficial veins? the largest of which appeared to empty into the latera sinus.

A right lateral craniotomy was carried out by Mr. Murray Falconer in the Dunedin Hospital and the right middle cerebral artery was clipped just proximal to the malformation. In his operation note he said that on opening the dura at the hinder part of the Sylvian fissure the venous side of the malformation was exposed at the surface. This was a large venous channel which coursed downwards and backwards over the upper aspect of the temporal lobe towards the lateral sinus and was about $2 \mathrm{~cm}$ long with a diameter of about $1 \mathrm{~cm}$. On splitting open the Sylvian fissure the middle cerebral artery was seen to be about 5 to $6 \mathrm{~mm}$ in diameter, running backwards over the insula for several centimetres until it turned sharply upwards to the parietal operculum. In the first part of its course the artery gave off at least one branch which crossed upwards over the insula. The arteriovenous malformation was mainly in the upper lip of the operculum; the venous head was lower than the point at which the artery entered the malformation. When the artery was clipped the large vein became slack and pulsation in it ceased. Small arteries coming round the edge of the operculum were pulsating and evidently received their blood supply from the middle cerebral artery before the point at which it had been clipped. Behind the venous part of the malformation was a cyst about $2 \mathrm{~cm}$ in diameter filled with subarachnoid fluid. This had evidently been the site of a 
previous bleed but at the operation no blood was found in its neighbourhood.

After recovery from the operation the patient was able to do her housework reasonably well but rather slowly and she remained free of epileptic attacks, although she showed confusion and was unable to do simple arithmetical problems.

Some nine years later she was admitted to Orokonui Home, confused, disorientated, euphoric, and with ideas of persecution. She behaved in an irresponsible and dangerous manner but responded well to tranquillizers. She remained in this condition for some six years. She was always normotensive. After being confined to bed for a few days with a severe urinary infection she died suddenly at the age of 59 .

At necropsy there was a massive pulmonary embolus which had followed deep calf vein thrombosis. Her large heart weighing $500 \mathrm{~g}$ showed a marked degree of hypertrophy of the left ventricle, which measured $1.8 \mathrm{~cm}$ in basal thickness. The kidneys were smooth and weighed 180 and $140 \mathrm{~g}$. There was a suppurative cystitis. There were no other significant findings apart from the brain and its blood vessels.

Post-mortem arteriography of the carotid and vertebral arteries (Fig. 1) showed that some of the medium injected intra-arterially had passed through the arteriovenous anastomosis into the superior anastomotic veins which drained into and filled the superior sagittal sinus with medium. The right lateral and sigmoid sinuses and the right internal jugular vein were also filled with the medium.

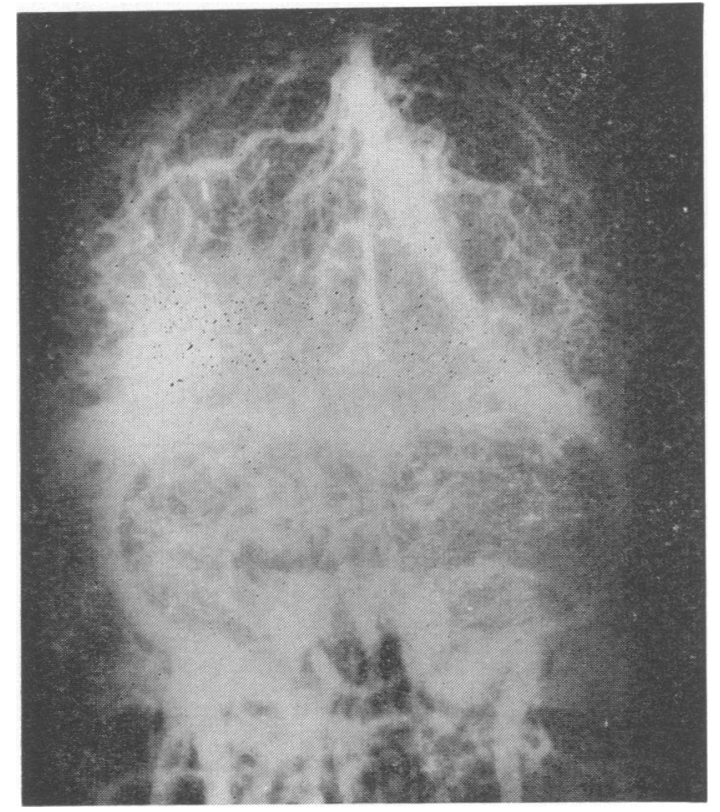

FIG. 1. Case 1. Post-mortem arteriogram showing a large right middle cerebral artery supplying the arteriovenous malformation from which large veins filled with medium drain into the sagittal and right lateral sinuses.
The brain and the neck vessels were removed and studied in detail after fixation. The carotid and vertebral arteries and the vessels of the circle of Willis and their major branches showed occasional minute atherosclerotic lipid plaques but no luminal narrowing. There were two right posterior cerebral arteries, one of which arose from the right internal carotid artery $5 \mathrm{~mm}$ distal to the posterior communicating artery. The right middle cerebral artery was a large vessel with a lumen $3 \mathrm{~mm}$ in diameter running directly to the grossly thickened leptomeninges over the lateral sulcus to supply a large mass of vessels in the posterior part of the insula.

Serial coronal slices of the cerebral hemispheres showed the right middle cerebral artery running backwards into the anterior end of the arteriovenous anastomosis in the lateral sulcus at a level anterior to the thalamus. The mass of the anastomosis extended posteriorly for about $3 \mathrm{~cm}$ and its widest part was approximately $2.5 \mathrm{~cm}$ in diameter. Within the mass of the anastomosis and almost $1 \mathrm{~cm}$ from the anterior end were three blackened silver clips which seemed still completely closed on a vessel. Posteriorly the mass of the anastomosis was separated from the dilated lateral ventricle only by ependyma-covered scar (Fig. 2).

Most of the white matter of the right temporal lobe and some cortex as well had been destroyed by an old haemorrhage with a resultant large orange-coloured contracted cavity running forward and replacing some of the cortex of the insula, the claustrum, the external capsule, the lateral part of the putamen, and ending in the right centrum semi-ovale and the white matter beneath the inferior frontal gyrus. The right lateral ventricle was also stained orange-brown.

Histological study showed the anastomosis to comprise many large arteries and veins distended by medium and lying in scar. There were a number of old occluded arteries (Fig. 3) but significantly fewer old occluded veins (Fig. 4). Some of the arteries showed almost complete recanalization, but no recanalized veins could be recognized.

\section{CASE 2}

T.V., a 46-year-old man, first presented with bilateral claudication and pain in the calves even at rest. Spinal anaesthesia and bilateral sympathectomies produced little relief. At the age of $\mathbf{4 9}$ he had a blood pressure of $190 / 80 \mathrm{~mm} \mathrm{Hg}$ and good posterior tibial and dorsalis pedis pulses were noted. At the age of 58 his blood pressure was $210 / 110 \mathrm{~mm} \mathrm{Hg}$. Some two weeks after this examination he was admitted to the Dunedin Hospital (28274) with paralysis of his right arm which had developed over one week. Although he also had a mild dysarthria, a diagnosis of 'hysterical monoplegia' was applied. Two months later he was again admitted to that hospital with a right hemiplegia and dysarthria.

At the age of 61 he had two episodes of weakness of the left arm lasting one day. Some months later he was admitted to the Dunedin Hospital with melaena and a major haematemesis. On examination he was unconscious with bilateral plantar extensor responses and his eyes were deviated to the right. Following treatment for his bleeding peptic ulcer he had aphasia, bilateral hemi- 


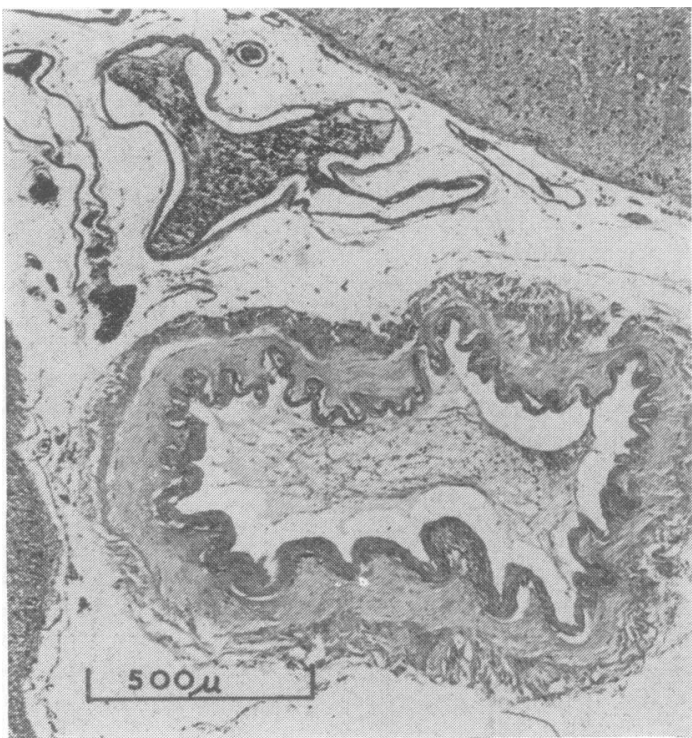

FIG. 3. Case 1. Photomicrograph of occluded artery and efferent vein containing injected medium in the arteriovenous malformation. The artery is occluded by organized fibrous tissue, the result of the surgical ligation 15 years previously. Haematoxylin and eosin, $\times 56$.

plegia, hemianopia, and a pseudo bulbar palsy. Ten months later at the age of 62 he died with a bronchopneumonia.

At necropsy the significant findings outside the head and neck were a bronchopneumonia, the scar of an old healed duodenal ulcer, and finely granular kidneys weighing 140 and $130 \mathrm{~g}$ with marked arteriolosclerosis
FIG. 2. Case 1. Coronal slice of brain at level of pineal showing mass of arteriovenous malformation with cavitation and staining resulting from old haemorrhage.

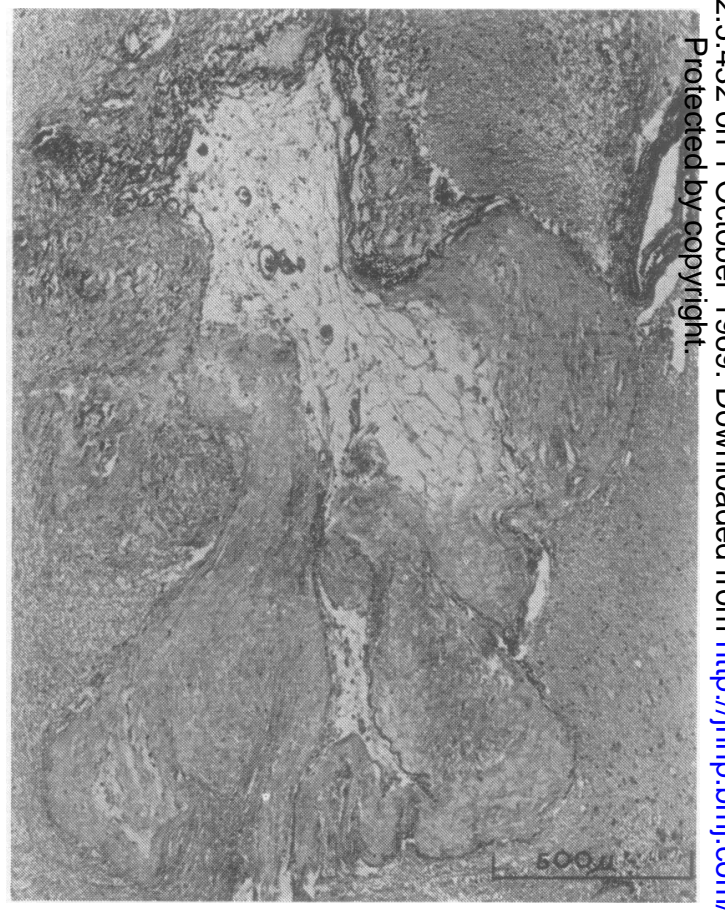

FIG. 4. Case 1. Photomicrograph of collapsed occluded 윽 vein in the arteriovenous malformation. The vein is occluded by organized fibrous tissue. Van Giesen elastic, $\times 56$.

suggesting hypertension, although the $340 \mathrm{~g}$ heart did $N$ not show any degree of left ventricular hypertrophy.

Post-mortem arteriography of the carotid and vertebral systems was performed and the entire carotid and 
vertebral artery system was further studied after fixation.

Old organized thrombus filled each internal carotid artery extending on the left to just beyond the dura and on the right into the first part of the right middle cerebral artery to the first major branch. Both ophthalmic arteries were occluded at the internal carotids. The only arterial supply from the circle of Willis to the anterior and middle cerebral arteries was by the small posterior comm-unicating arteries, each of which had a lumen of only $0.5 \mathrm{~mm}$ diameter even when distended with injection medium. Both vertebral arteries had a number of atherosclerotic plaques but the medium-distended lumina were 3 to $5 \mathrm{~mm}$ in diameter.

The brain weighed only $940 \mathrm{~g}$ and showed a number of large thin-walled venous channels filled with medium. on the surface of the right side which drained into the venous sinuses (Fig. 5). There was a very large tortuous channel running on the superior surface of the right temporal lobe, over the anterior tip, and back along the under surface of the lobe to drain into the lateral sinus. A branch of this ran from the superior surface of the temporal lobe to the spheno-parietal sinus, while an extension ran over the right parieto-frontal area to drain into the superior sagittal sinus. The venous sinuses and both internal jugular veins were filled with medium. These large venous channels drained a plexus of small vessels on the inferior and lateral surfaces of the right temporal lobe (Fig. 6) which was fed by branches of the right posterior cerebral artery, by branches of the right middle cerebral artery which was patent and filled with medium from the first branch distally and by branches of the right anterior cerebral artery. There was an old large infarct of the posterior central part of the left middle cerebral artery territory and a massive old infarct involving most of the right middle cerebral artery territory and the central part of the right anterior cerebral artery territory (Fig. 7). As well, there was a small cystic infarct in the anterior part of the left centrum semi-ovale.

Histological study of the two large cerebral infarcts

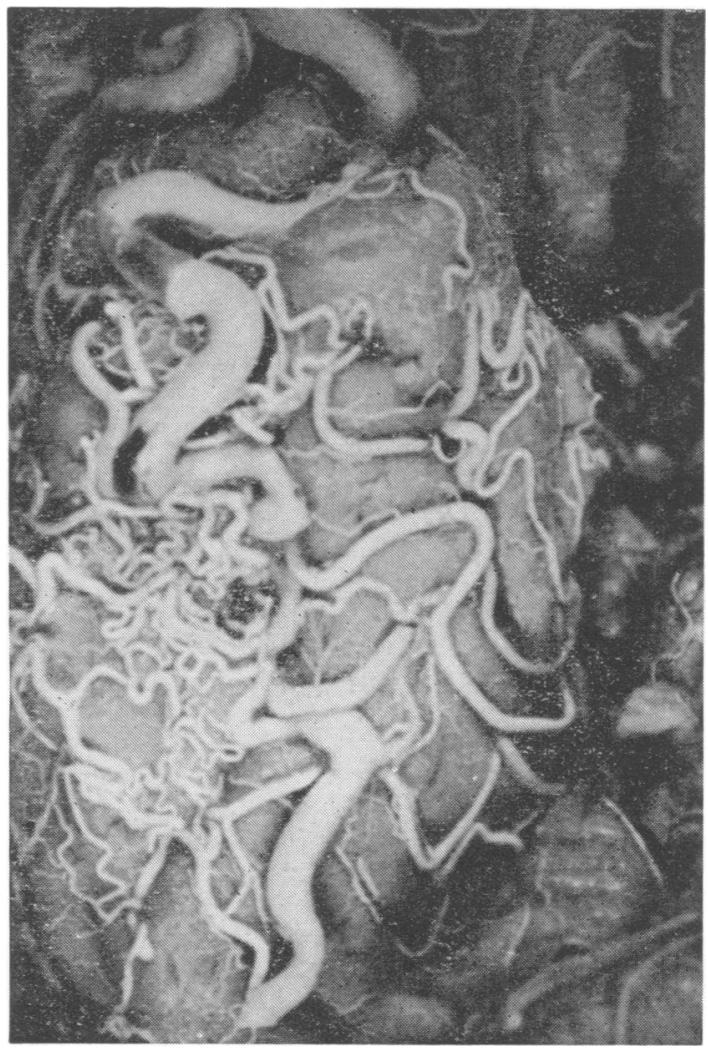

FIG. 6. Case 2. Inferior aspect of right temporal lobe showing plexus of injected arteries which are fed by posterior, middle, and anterior cerebral artery branches. Some of the injection medium has been inadveretntly squeezed out of the large veins draining the arteriovenous malformation.

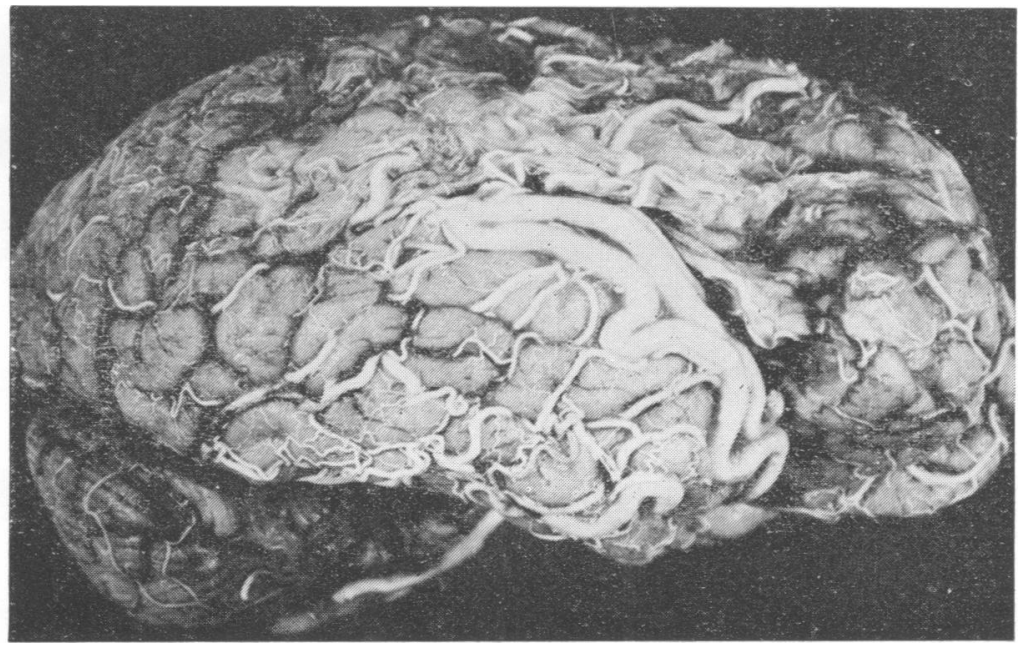

FIG. 5. Case 2. Right side of brain showing large thin-walled veins distended by medium injected intra-arterially, lying on surface of temporal lobe and draining into the lateral and sphenoparietal sinuses and on surface of parietofrontal region and draining into the superior sagittal sinus. There is gross shrinkage of the area involved by the right middle cerebral artery territory infarct. 


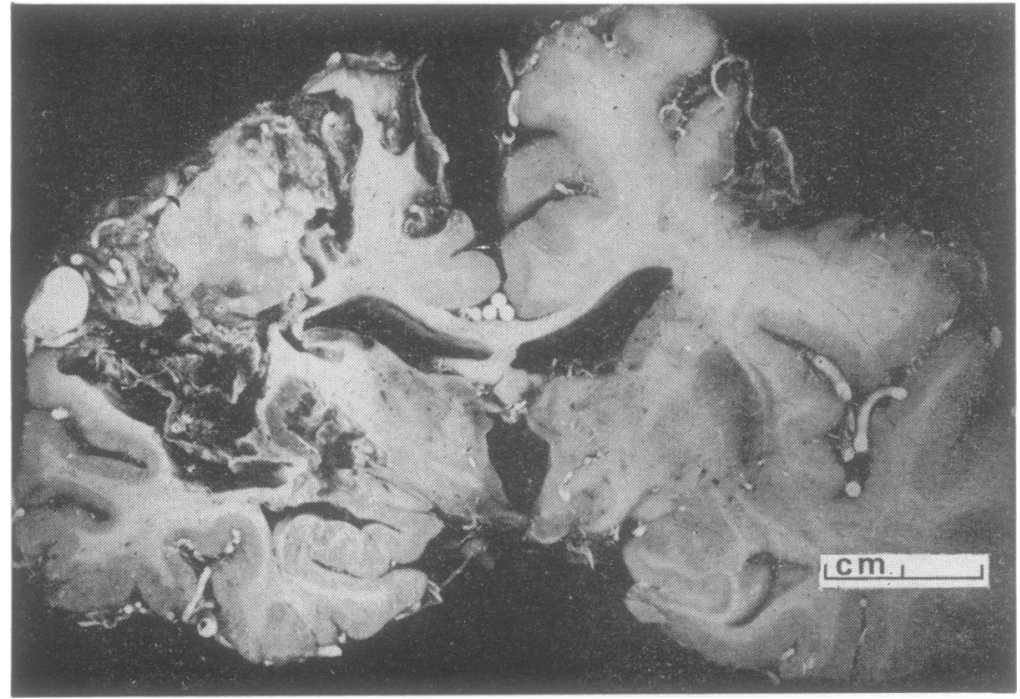

FIG. 7. Case 2. Coronal slice of brain at level of the thalamus showing some of the efferent veins of the arteriovenous malformation and the quite massive bilateral old middle cerebral artery territory infarcts.

showed extensive gliosis with recanalization of many meningeal arteries (Fig. 8). Sections of the pons and medulla showed almost complete degeneration of the right and appreciable degeneration of the left corticospinal tracts. Sections of the efferent veins of the anastomosis showed rather more muscle in the wall than normal (Fig. 9).

In summary (Fig. 10), there was a right cerebral arteriovenous anastomosis with afferent components from the right anterior, middle, and posterior cerebral arteries and efferent components to the sphenoparietal, superior sagittal, and lateral venous sinuses. The atherosclerotic left internal carotid artery was occluded by an old organized thrombus and there was a large 4-year-old infarct in the left middle cerebral artery territory. The atherosclerotic right internal carotid artery was occluded by an old organized thrombus which extended into the middle cerebral artery to the first main branch. There was a massive 10-month-old infarct involving most of the right middle cerebral artery territory and the central part of the right anterior cerebral artery territory. There was a small old cystic infarct in the left anterior centrum.

\section{DISCUSSION}

The usual features of the common form of cerebral arteriovenous malformation (Dandy, 1928; Mackenzie, 1953; Tönnis, Schiefer, and Walter, 1958; Perret and Nishioka, 1966) are demonstrated in the first case. The lesion was a localized mass of vessels in the parietal region fed by branches of the internal carotid artery and the patient presented around the age of 30 with subarachnoid haemorrhage, had headaches, epileptic seizures and a hemiparesis and subsequently developed mental deterio-

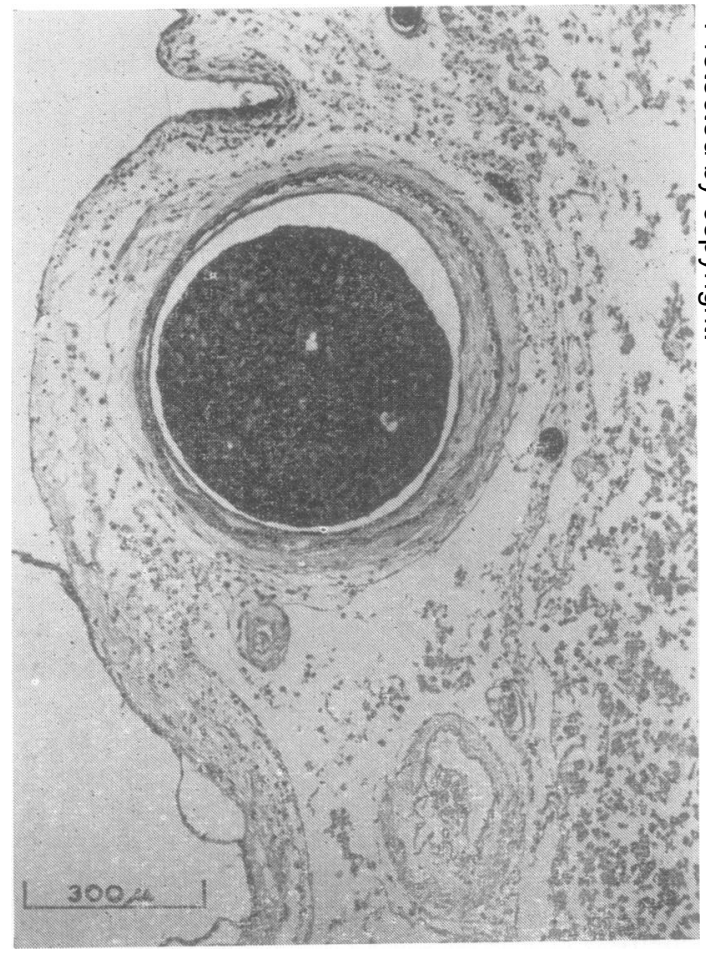

FIG. 8. Case 2. Photomicrograph showing large, almost fully recanalized artery and small partially recanalized artery both containing injection medium in the leptomeninges overlying the old right middle cerebral artery territory infarct. Haematoxylin and eosin, $\times 75$. 


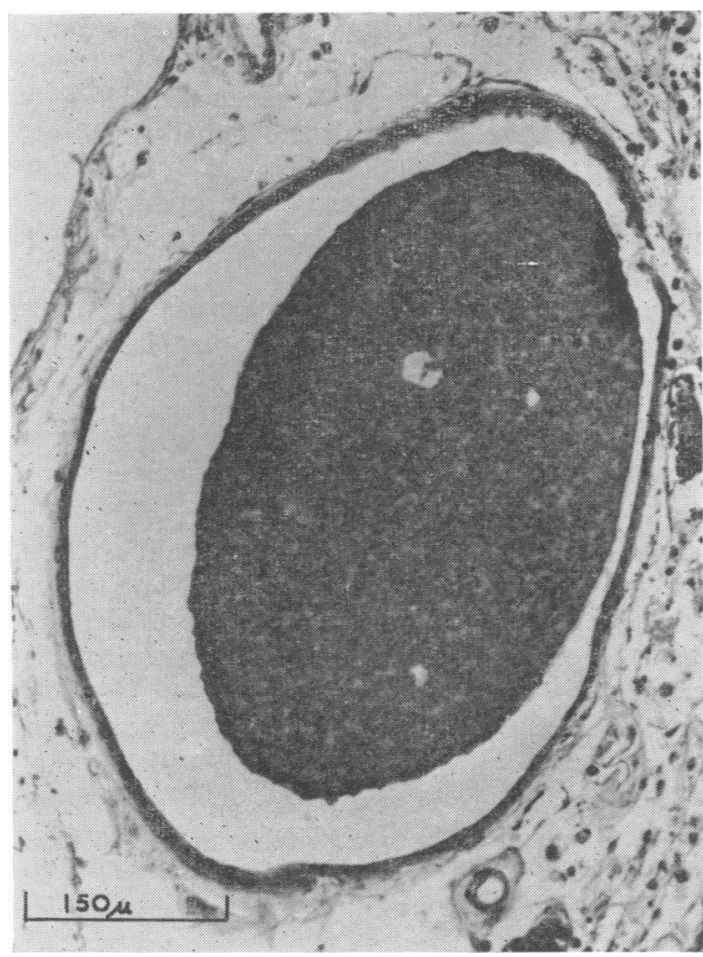

FIG. 9. Case 2. Photomicrograph of somewhat arterialized meningeal vein efferent from the arteriovenous malformation and thus distended with injection medium. Some of the compound granular corpuscles and debris of the underlying right-sided infarct are shown. Haematoxylin and eosin, $\times 190$.

ration. The attempt at surgical treatment was made by clipping the largest afferent artery as it entered the lesion, but at necropsy some 15 years later the lesion had enlarged and the clips were then situated within the lesion. Walker (1956) has argued against significant progression of arteriovenous malformations, but Cushing and Bailey (1928), Olivecrona and Riives (1948), Höök and Johanson (1958), Anderson and Korbin (1958) cite many examples of striking progression. Although it could be assumed that silver clips on arteries might in time gradually open, the clips in this case remained closed even after 15 years and the enlargement of the malformation resulted from its natural progression with the widening of anastomotic afferent arterial channels, developed by the use of other and alternative middle cerebral artery branches after prolonged increased pressure gradients along them.

A feature of the vascular anomaly in the second case is the large mass of dilated tortuous venous channels suggesting the lesion might be regarded
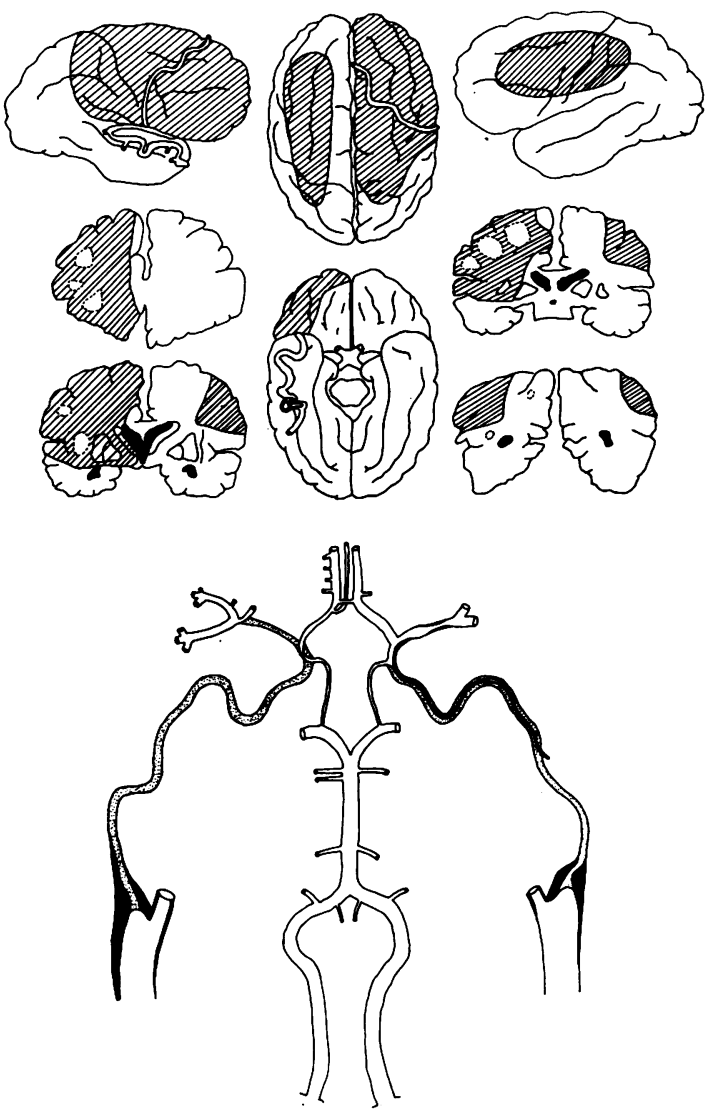

FIG. 10. Case 2. Diagram of major carotid-vertebralcerebral artery pattern and cerebral ischaemic lesions (shaded). Both internal carotid arteries are occluded by shrunken organized thrombi (stippled) with some recanalization on the left. The proportional pathways of the afferent arterial contributions to and the major efferent venous drainage from the vascular malformation are illustrated.

as a so-called venous racemose angioma (Olivecrona and Ladenheim, 1957), venous angioma (Zülch, $1965)$ or venous malformation (Russell and Rubenstein, 1959; McCormick, 1966). Nevertheless Olivecrona and Ladenheim consider 'a predominant venous hue' necessary to distinguish this ill-defined entity in the brain. As shown in Fig. 6 the intraarterial injection medium fills a small plexus of afferent arterial channels and has passed into the efferent veins so that the lesion is undoubtedly an arteriovenous malformation. Further, the necropsy injection technique employed enabled a number of arterial branches to be specifically traced directly into large veins and, using this criterion, the lesion could also be classified as an anomalous arterio- 
venous aneurysm (Olivecrona and Ladenheim, 1957), but such categorization seems confusing, as any vascular malformation is anomalous. To enable the variations of the vascular patterns in these lesions to be more clearly defined, it appears that injection studies accurately delineating the vascular channels need to be made in many more cases.

Feindel and Perot (1965) have described red arterial blood in the veins draining cerebral infarcts due to inadequate oxygen utilization by the necrotic tissue and Lassen (1966) has called this phenomenon the luxury-perfusion syndrome. This has been ascribed to a regional failure of the normal vascular autoregulation about the affected area of brain most likely resulting from an acute metabolic acidosis (Høedt-Rasmussen, Skinhoj, Paulson, Ewald, Bjerrum, Fahrenkrug, and Lassen, 1967). Rowbotham and Little (1965) have nevertheless suggested this localized over-abundant cerebral blood flow may be due to the opening up of small leptomeningeal arteriovenous shunts and also that abnormalities of these shunts may develop into large arteriovenous lesions. Is it possible that the arteriovenous anastomosis in the second case might have developed from normal vessels after the occurrence of the right cerebral infarct?

The meningeal arteries overlying old infarcts are frequently recanalized (Winter and Gyori, 1960; Romanul and Abramowicz, 1964). Dible (1958) has shown that recanalization is the process of reforming through channels and seems to be entirely confined within the vessel or its branches (Akrawi and Wilson, 1950), so that it seems possible for an arteriovenous anastomosis to develop in relation to an infarct only if the recanalization could involve an existing arteriovenous channel. For this reason, it is unlikely that the arteriovenous aneurysm in case 2 could have developed from normal leptomeningeal arteriovenous shunts. Further, the main mass of the arteriovenous anastomosis was over the temporal lobe and only its periphery was related to the infarct. Also the clinical history of the left hemiplegia some 10 months before death accurately dates the occurrence of the right cerebral infarction, and the extensive right cerebral arteriovenous anastomosis is much too large to have developed in this short period. It must be concluded that the lesion is a malformation which was already present when infarction happened in that hemisphere. The infarct apparently resulted from the right internal carotid thrombosis, but it may be that the abnormal blood flow through the arteriovenous malformation contributed to the ischaemic necrosis in the right hemisphere. Although gross neurological deficit due to haemorrhage from an arteriovenous malformation is common, complicating massive cerebral infarct is not referred to even in the reviews of large series of cases (Svien and McRae, 1965; Perret and Nishioka, 1966). Massive cerebral infarct seems indeed a remarkably rare complication of arteriovenous malformation.

Perret and Nishioka (1966) indicate that most authorities agree that internal carotid ligation is an ineffective treatment for cerebral arteriovenous malformations. Although the size of the malformation in case 2 was unknown till necropsy, it seems unlikely that it was significantly altered by the thrombotic internal carotid occlusion some 10 months before death.

\section{SUMMARY}

Two cases of cerebral arteriovenous malformation are reported in which post-mortem arteriography was used as an aid to the necropsy study of the vascular pattern. The first case had been treated by surgical clipping of the afferent artery some 15 years before death and demonstrated measurable progression of the lesion with clinical deterioration in that period. The second case presented bilateral internal carotid occlusion and bilateral massive cerebrato infarcts. The possible relationships of the malformo ation to the ischaemic lesions is discussed.

We acknowledge the assistance provided at the Uni versity of Otago by the New Zealand Medical Research Council and we are grateful to Mr. Murray Falconer foo permission to quote from his operation notes in case We also thank Dr. A. C. Begg and his radiographif. staff for the radiographs, Mr. D. Tingle for the histo logical preparations, and Mr. S. Mathers for help with 6 the photographs.

\section{REFERENCES}

Akrawi, Y. Y., and Wilson, G. M. (1950). Observations on the development and function of elastic-coated vascular channels in occluded arteries. J. Path. Bact., 62, 69-73.

Anderson, F. M., and Korbin, M. A. (1958). Arteriovenous anomalies of the brain; a review and presentation of 37 cases. Neurology (Minneap.), 8, 89-101.

Cole, F. M., and Yates, P. O. (1967). The occurrence and significance of intracerebral micro-aneurysms. J. Path. Bact., 93, 393-411.

Cushing, H., and Bailey, P. (1928). Tumours Arising from the BloodVessels of the Brain; Angiomatous Malformations and Hemangioblastomas. Thomas: Springfield, Ill.

Dandy, W. E. (1928). Arteriovenous aneurysm of the brain. Arch. Surg. (Chic.), 17, 190-243.

Dible, J. H. (1958). Organisation and canalisation in arterial thrombosis. J. Path. Bact., 75, 1-7.

Feindel, W., and Perot, P. (1965). Red cerebral veins: a report on arteriovenous shunts in tumors and cerebral scars. J. Neurosurg., 22, 315-325.

Høzdt-Rasmussen, K., Skinhoj, E., Paulson, O., Ewald, J., Bjerrum, J. K., Fahrenkrug, A., and Lassen, N. A. (1967). Regional cerebral blood flow in acute apoplexy. The "luxury-perfusion syndrome' of brain tissue. Arch. Neurol. (Chic.), 17, 271-281.

Höök, O., and Johanson, C. (1958). Intracranial arteriovenous aneurysms; a follow-up study with particular attention to their growth. Arch. Neurol. Psychiat. (Chic.), 80, 39-54.

Hutchinson, E. C., and Yates, P. O. (1957). Carotico-vertebral stenosis. Lancet, 1, 2-8. 
Johnson, R. T., and Yates, P. O. (1956). Brain stem haemorrhages in expanding supratentorial conditions. Acta. radiol. (Stockh.), 46, 250-256.

Kaplan, H. A., Aronson, S. M., and Browder, E. J. (1961). Vascular malformations of the brain. An anatomical study. J. Neurosurg., $18,630-635$.

Lassen, N. A. (1966). The luxury-perfusion syndrome and its possible relation to acute metabolic acidosis localised within the brain. Lancet, 2, 1113-1115.

McCormick, W. F. (1966). The pathology of vascular ('arteriovenous') malformations. J. Neurosurg., 24, 807-816.

Mackenzie, I. (1953). The clinical presentation of cerebral angioma: review of 50 cases. Brain, 76, 184-214.

Olivecrona, H., and Ladenheim, J. (1957). Congenital Arterioverous Aneurysms of the Carotid and Vertebral Arterial Systems. Springer: Berlin.

_- , and Riives, J. (1948). Arteriovenous aneurysms of the brain; their diagnosis and treatment. Arch. Neurol. Psychiat. (Chic.), 59, 567-602.

Perret, G., and Nishioka, H. (1966). Report on the co-operative study of intracranial aneurysms and subarachnoid haemorrhage. Sect. VI. Arteriovenous malformations. An analysis of 545 cases of cranio-cerebral arteriovenous malformations and fistulae reported to the co-operative study. J. Neurosurg., $25,467-490$.

Rodda, R. A., and Dugdale, L. M. (1963). The necropsy demonstration of carotid, vertebral and cerebral artery disease in cases of cerebral infarct. N.Z. med.J., 62, 510-519.
Romanul, F. C. A., and Abramowicz, A. (1964). Changes in brain and pial vessels in arterial border zones: a study of 13 cases. Arch. Neurol. (Chic.), 11, 40-65.

Rowbotham, G. F., and Little, E. (1965). A new concept of the circulation and the circulations of the brain: the discovery of surface arteriovenous shunts. Brit. J. Surg., 52, 539-542.

Russell, D. S., and Rubenstein, L. J. (1959). Pathology of Tumours of the Nervous System, pp. 88-89. Arnold: London.

Russell, R. W. R. (1963). Observations on intracerebral aneurysms. Brain, 86, 425-442.

Stein, B. M., McCormick, W. F., Rodriguez, J. N., and Taveras, J. M. (1962). Post mortem angiography of cerebral vascular system. Arch. Neurol. (Chic.), 7, 545-559.

Svien, H. J., and McRae, J. A. (1965). Arteriovenous anomalies of the brain: fate of patients not having definitive surgery. J. Neurosurg., 23, 23-28.

Tönnis, W., Schiefer, W., and Walter, W. (1958). Signs and symptoms of supratentorial arteriovenous aneurysms. J. Neurosurg., $15,471-480$.

Vander Eecken, H. M., and Adams, R. D. (1953). The anatomy and functional significance of the meningeal arterial anastomoses of the human brain. J. Neuropath. exp. Neurol., 12, 132-157.

Walker, A. E. (1956). Clinical localization of intracranial aneurysms and vascular anomalies. Neurology (Minneap.), 6, 79-90.

Winter, W. J., and Gyori, E. (1960). Pathogenesis of small cerebral infarcts. Arch. Path. (Chic.), 69, 224-234.

Zülch, K. J. (1965). Brain Tumours: Their Biology and Pathology, p. 248 (2nd American edn. translated by A. B. Rothballer and J. Olszewski). Springer: New York. 